\title{
Amount of maternal factor within the male pronucleus affects the reprogramming and developmental efficiency of androgenetic embryos
}

\section{Chen Liao}

Harbin Medical University

\section{Xinghui Shen}

Harbin Medical University

Yuwei Zhang

Harbin Medical University

Lei Lei ( $\square$ lei086@ems.hrbmu.edu.cn )

https://orcid.org/0000-0001-5372-8749

\section{Research article}

Keywords: Reprogramming, androgenetic embryos, paternal pronucleus, maternal materials, Tet3, H3.3, pronucleus transfer, double sperm injection

Posted Date: August 23rd, 2019

DOI: https://doi.org/10.21203/rs.2.13508/v1

License: (c) (i) This work is licensed under a Creative Commons Attribution 4.0 International License. Read Full License 


\section{Abstract}

Background Uniparental embryos have uniparental genomes and are very useful models for studying parental specific gene expression or for exploring the biological significance of genomic imprinting in mammals. However, the early developmental efficiency of androgenetic embryos is significantly lower than that of parthenogenetic embryos. In addition, oocytes are able to reprogram the nuclei of sperm after fertilization to guarantee embryonic development by maternal derived reprogramming factors, which accumulate during oogenesis. However, importance of maternal materials in the efficiency of reprogramming the pronucleus of androgenetic embryos has not been ascertained.Results Androgenetic embryos were constructed artificially by pronucleus transfer (PT) or double sperm injection (DS) in our experiments. Compared with the androgenetic embryos constructed artificially by DS, those constructed by PT, which derived from two zygotes, contained more maternal material (like Tet3 and H3.3). This study confirmed the better developmental potential of PT embryos, with higher blastocyst rates, the stronger expression of pluripotent genes, the lower expression of apoptotic genes, and superior blastocyst quality.Conclusions The aggregation of more maternal materials in the paternal pronucleus facilitated the reprogramming of the paternal genome, improving embryonic development in pronucleus transfer androgenesis.

\section{Background}

Mammalian uniparental embryos only contain either paternal or maternal genomes, and are extremely difficult to develop into individuals. However, Kono et al. [1] regulated the expression of imprinted genes in mice parthenogenetic embryos, and successfully obtained viable individuals in 2004. More recently, Li et al. [2] derived androgenetic diploid embryonic stem cells (ESCs) from androgenetic diploid embryos by the co-injection of six imprinting gene knock-out androgenetic haploid ESCs (ahESCs) and a sperm. The authors attempted to produce bipaternal mice by tetraploid complementation, and produced 12 live fullterm bipaternal pups; however, the pups died within $48 \mathrm{~h}$ of birth, due to suckling and breathing deficiencies. Androgenetic embryos serve as ideal models for investigating paternal specific gene expression and the biological significance of imprinted genes. However, the developmental ability of androgenetic embryos is very poor [3]. Androgenetic development is limited by the aberrant expression of imprinted genes, inadequate reprogramming, and low developmental rate (at most $55 \%$ blastocyst rate) [4] versus $90 \%$ in parthenogenetic embryos [5].

Once the sperm enters the cytoplasm of the oocyte, the condensed paternal genomes (which are wrapped by protamine) are uncovered by the oocyte, and are transformed to a status of decompaction and accessibility by the replacement of histones [6]. Then, the paternal genomes are subject to a series of intricate reprogramming processes, spatially [7]. These processes contribute towards erasing epigenetic modifications that formed during spermatogenesis, regulating the initial expression of zygotic genomes correctly, and generating well-developed totipotent embryos. Reprogramming processes involve the demethylation of parental DNA, as well as post-translational modifications, including methylation, 
acetylation, and the phosphorylation of the histone terminal, chromatin reorganization, and miRNA regulation.

Maternal gene-coded materials accumulate to meet the needs of meiosis, ovulation, and preimplantation development, before zygote genome activation (ZGA) [8]. Then, maternal material enters the paternal pronucleus and reprograms paternal genomes that are important for ZGA and subsequent development [9]. Maternal H3.3 (histone variant 3.3) [10] is a substitute protamine in the sperm nucleosome that facilitates paternal genome reprogramming and maintains the decondensation state of chromatin. It is an important reprogramming-related factor for activating various pluripotent genes. Knocking out $\mathrm{H} 3.3$ in oocytes impairs the formation of the paternal pronucleus, DNA replication, and rDNA transcription [11]. After fertilization, the oxidation of 5-methylcytosine $(5 \mathrm{mC})$ mediated by Tet3 (ten-eleven translocation DNA dioxygenase 3) facilitates DNA demethylation and activation of the paternal genome $[12,13]$.

The developmental rate of androgenetic embryos constructed by DS (double sperm injection) to form enucleated oocytes is quite low, with just $20 \%$ of embryos developing to blastocysts [14]. In comparison, androgenetic embryos derived from the PT (pronucleus transfer) of two zygotes exhibit higher developmental rates to blastocysts (55\%) [4]. Liu et al. [15] found that the reprogramming capacity of the parental pronuclei varies, with crucial reprogramming factors being primarily located in paternal pronuclei.

Based on existing studies, in our study, we chose H3.3 and Tet3 as the detection indicators. we hypothesized that PT androgenetic embryos contain more maternal factors (such as H3.3 and Tet3) that are required for reprogramming compared to DS androgenetic embryos, resulting in much higher developmental rate and better quality PT embryos. This study will advances our understanding of the effect of maternal factors on the activation of sperm-derived paternal genome.

\section{Results}

\section{Differences in developmental efficiency between two types of androgenetic embryos}

For DS embryos, two sperm heads were injected into the cytoplasm of one oocyte, from which the spindle-chromosome complex was removed, after artificial activation. As a result, one integrated pronucleus (rather than two individual pronuclei) developed in the DS embryo (Fig. 1A). However, in a PT embryo derived from two zygotes, one maternal pronucleus became depleted, and one paternal pronucleus from the other zygote was translocated to the zona pellucida with an unbroken plasma membrane to ensure successful electrofusion (Figure. 1B).

Firstly, we examined the developmental rates of PT and DS preimplantation embryos in vitro. The fertilized embryos derived from ICSI (normal control) had the highest blastocyst rate (68.9\%). For the androgenetic embryos, PT embryos had significantly higher 4-cell and blastocyst rates ( $86.4 \%$ and $50.0 \%)$ 
compared to DS embryos (72.0\% and 7.6\%) (Table 2). Thus, androgenetic embryos had lower developmental rates compared to ICSI embryos; however, PT embryos were more similar to ICSI embryos.

To compare the quality of androgenetic blastocysts, we first observed morphology of two types of androgenetic blastocysts at $108 \mathrm{~h}$ post fertilization. Expanded blastocyst cavities or hatched blastocysts were detected in the PT blastocyst. In comparison, the extension of DS blastocysts was not obvious (Fig. 2). In addition, we examined the number of cells in the inner cell mass (ICM) and trophoblast cells (TE) of androgenetic blastocysts using immunofluorescence. ICSI blastocysts were treated as the normal control. The average total number of cells and the ICM and TE ratios in PT blastocysts were much lower compared to those in ICSI blastocysts; however, the ratio of ICM to TE between PT and ICSI was comparative (0.42 vs 0.45 ) (Table 3 ). Moreover, the number of TE cells in PT and ICSI blastocysts far exceeded those in DS blastocysts (Table 3). Thus, the developmental efficiency of PT androgenetic embryos was better than that of DS embryos.

\section{Expression of H3.3 and Tet3 in pronucleus of androgenetic embryos}

To confirm whether more reprogramming related maternal factors accumulated in the paternal pronucleus of PT embryos, we examined H3.3 protein content in PT and DS embryos at $10 \mathrm{~h}$ post fertilization by western blotting. The paternal pronuclei of PT embryos contained the highest H3.3 levels compared to the DS group (Fig. 3). H3.3 levels in the maternal pronucleus transfer (MT) group showed that $\mathrm{H} 3.3$ accumulates in the paternal pronucleus, rather than the maternal pronucleus.

The expression level of the other maternal factor, Tet3, was also examined by immunofluorescence. Tet3 expression was similar to that obtained for H3.3 (Fig. 3). The PT group had the highest florescence intensity, while the MT group had the lowest (Fig. 4). Thus, reprogramming related maternal factors were mainly located in the paternal pronucleus.

\section{Demethylation level in two kinds of androgenetic embryos}

To investigate the degree of demethylation of androgenetic embryos, we examined $5 \mathrm{mC}$ and $5 \mathrm{hmC}(5-$ hydroxymethylcytosin) levels in PT and DS embryos at $10 \mathrm{~h}$ post fertilization. MT embryos served as the control. The fluorescence intensity of $5 \mathrm{hmC}$ in PT embryos significantly increased, while the intensity of $5 \mathrm{mC}$ in PT noticeably decreased, when compared with DS embryos. Moreover, there was no obvious discrepancy to signal intensity between MT and DS embryos. Thus, the extent of epigenetic reprogramming was higher in PT embryos, which might explain the better developmental efficiency (Fig. 5). 


\section{Expression of pluripotent and apoptotic genes in the two types of androgenetic embryos}

To verify the reprogramming efficiency and apoptosis in androgenetic embryos, PT and DS embryos were collected at 78, 96, $108 \mathrm{~h}$ post fertilization as morula, early blastocysts, and late blastocyst, respectively. We used ICSI embryos as the control. Next, we examined the expression of pluripotent markers Oct4, Nanog, Cdx2 and Gata4, and the apoptotic marker Casp3 through qPCR. In the DS group, there was a significant decline in the expression of Oct4, Nanog, Cdx2, and Gata4 in the androgenetic morulae (Fig. 6A). However, there was no obvious difference in the PT group compared to the ICSI group; however, Nanog mRNA decreased and Casp3 mRNA increased. At the early blastocyst stage, the levels of all pluripotent markers (except Oct4) were much lower in the DS group. In comparison, only Oct4 and Nanog were abnormally expressed in the PT group (Fig. 6B). For late blastocysts, the expression of Oct4 and Nanog notably increased in the DS group. Furthermore, the expression of Cdx2 and Gata4 was still lower in the DS group compared to the ICSI group. However, only Oct4 expression was slightly lower, while other pluripotent genes showed no obvious changes (Fig. 6C). Regardless of stage (i.e., morula stage, early and late blastocyst), DS embryos had the highest Casp3 expression levels, which might drive their low developmental efficiency. Thus, the expression of pluripotent and apoptotic genes was aberrant in DS embryos, while the genes of PT embryos were more similar to those of ICSI embryos.

\section{Discussion}

This study demonstrated that the pronuclei of PT embryos contain more maternal material and more efficient reprogramming compared with DS embryos, leading to significantly higher developmental efficiency.Furthermore, we confirmed that maternal materials are vital for reprogramming based on comparisons of two types of androgenetic embryos.

After fertilization, maternal materials accumulated in the cytoplasm of oocytes, contributing to the process of pronucleus formation by entering the pronucleus. Furthermore, in one DS embryo, two sets of paternal genomes shared maternal materials in a single enucleated oocyte. To generate a PT embryo, two paternal pronuclei (which were reprogrammed by adequate maternal materials in a single zygote, respectively) were placed in the same zygote in which the maternal pronucleus had been eliminated. After fertilization, maternal reprogramming-related materials preferentially entrance into the sperm-derived paternal nucleus, as previously demonstrated by Liu et al. [15].Therefore, we speculated that the maternal material that accumulates during oogenesis in an oocyte is not sufficient to reprogram two sets of paternal genomes. PT embryos clearly had superior blastocyst rates compared to DS embryos, confirming that maternal material is required to reprogram zygotes.

Moreover, previous studies have confirmed that maternal factors have diverse roles using mice genemodified models. These factors are involved in several aspects of early embryonic development, including the degradation of maternal mRNA, chromatin remodeling, and epigenetic modification. H3.3 and Tet3 are key maternal materials that are involved in the reprogramming and demethylation of the 
paternal genome, respectively. By examining H3.3 and Tet3 content in androgenetic embryo pronuclei, we showed that DS embryos had lower H3.3 and Tet3 protein levels. Epigenetic reprogramming (such as DNA demethylation) was closely associated with the regulation of gene expression during preimplantation development. Active and rapid DNA demethylation was documented in the paternal pronucleus of the zygote. In comparison, replication-dependent passive DNA demethylation in the maternal pronucleus took place. $5 \mathrm{hmC}$ was the intermediate state of demethylation, which was oxidized from $5 \mathrm{mC}$, and serves as a key indicator for demethylation. In addition, $5 \mathrm{hmC}$ and Tet 3 have significant roles in reprogramming after fertilization [11]. In the current study, $5 \mathrm{hmC}$ levels were lower in the DS group, similar to that of Tet3, indicating insufficient demethylation in DS embryos. This phenomenon might give rise to the much lower development efficiency directly compared with PT in this study. Previous studies of mice also indicated that the developmental rate of PT androgenetic embryos is significantly higher than that of DS embryos $[4,14]$. Incidentally, parthenogenetic embryos had extremely high preimplantation developmental rates, probably because of the low methylation level in maternal pronuclei, which easily reached reprogramming destinations.

The extent of reprogramming influences the activation of the embryonic genome and embryonic development directly and, thus, the expression of pluripotent genes. Four pluripotent markers (Oct4, Nanog, Cdx2, and Gata4) were selected for this study. Oct4 and Nanog are involved in regulating the gene expression of embryonic stem cells, and impacts pluripotency and the self-renewal capacity of cells [16]. The transcription of Oct4 begins at the 4-cell stage in embryos, at which point expression peaks in the inner cell mass [17]. In comparison, the morula stage is the earliest phase at which transcription is detected for Nanog [18]. Cdx2 is a critical transcriptional factor that is involved in inducing differentiation in blastocyst trophectoderm cells, which are mainly expressed in the outer cells of the morula and the nuclei of trophectoderm cells [19]. Gata4 is the key factor associated with the formation of endocardium and the regulation of cardiogenesis, which is expressed in the primitive endoderm [20]. The similar expression of the pluripotent genes of PT embryos with those of ICSI embryos in our study might explain the higher developmental efficiency of PT embryos. However, the abnormal expression of Oct4 and Nanog might be caused by delayed development and gene expression; in other words, the gene expression of DS embryos is in a growing phase, whereas those in PT embryos has already reached a high and steady level.

This study focused on the preimplantation development of mice embryos. However, the postimplantation development of these two types of androgenetic embryo also needs to be elucidated. This study showed that the number of TE cells in PT embryos exceed those in DS embryos, allowing us to proceed to this next step. Furthermore, mechanisms, other than the imprinting defects identified by Li et al. [2] might contribute to androgenetic reproduction, and need to be identified. In future, knockdown H3.3 or Tet3 in androgenetic embryos could help confirm the function of these two maternal materials. How other maternal factors function in the reprogramming process should also be explored. On the other side, this study did not focus on the regulation of imprinted regions, next step we will attempt to produce androgenetic mice by controlling the expression of maternal or paternal imprinted genes. 
In the future, this research advances our understanding of the regulation and function of maternal factors on zygotic genome activation, providing a basis for developing new methods of examining reproduction.

\section{Conclusion}

We revealed that the pronuclei in PT embryos possessed more maternal materials and more sufficient reprogramming, consequently obtained significantly higher developmental efficiency.Further, maternal materials were proved to be vital for reprogramming through comparing two kinds of androgenetic embryos.

\section{Methods}

\section{Animals}

B6D2F1 (DBA/2 × C57BL/6) male/female mice were purchased at 7-10 weeks in age from Beijing Vital River Laboratory Animal Technology Co., Ltd, Beijing, China. The mice were kept in clean cages, and the surrounding temperature was maintained between $17^{\circ} \mathrm{C}$ and $22^{\circ} \mathrm{C}$. The lights were turned on from 5:00 to 19:00. All animal experiments were implemented in line with the Guidelines for Animal experiments of the Harbin Medical University (HMUIRB20190011). The approaches were approved by Harbin Medical University Ethics Committee. Unless stated, all reagents for embryo culture were purchased from Sigma (USA).

\section{Oocyte and zygote collection and spermatozoa preparation}

Female B6D2F1 mice were superovulated by injecting 5IU pregnant mare serum gonadotropin (PMSG, $\mathrm{NSH}$, China), followed by 5IU human chorionic gonadotropin (hCG, NSH, China) $48 \mathrm{~h}$ later. To obtain zygotes, female mice were mated with male B6D2F1 mice after hCG injection. Approximately $14 \mathrm{~h}$ and 17 $\mathrm{h}$ after HCG injection, the oocytes and zygotes were collected from the fallopian tubes. Mice were anesthetized by 2,2,2-tribromoethanol $(125 \mathrm{mg} / \mathrm{kg})$, and then euthanized by carbon dioxide suffocation after collection. Corpse were frozen for uniform disposal. Cumulus-free oocytes with appropriately sized perivitelline space and homogeneous ooplasm were selected, while only zygotes with two distinct parental pronuclei were selected. They were placed in $20 \mu l$-droplets of CZBG, and covered by sterile mineral oil (Fisher, $0121-20$ ). Then, the oocytes were placed in an incubator set to $37^{\circ} \mathrm{C}$ and $5 \% \mathrm{CO}_{2}$ until use. Spermatozoa were collected from the cauda epididymis of male B6D2F1 mice at 8-10 weeks in age. The spermatozoa were then placed in CZB-HEPES medium until injection.

\section{Generation of ICSI, PT, and DS embryos}

ICSI, implemented by a piezo-driven unit following a previously described method [21], was treated as control; however, our experiments were performed in HEPES-CZB containing $5 \mu \mathrm{g} / \mathrm{ml}$ cytochalasin B 
(Sigma, C6762) at room temperature. The ICSI-generated embryos were washed at least three times, and were placed in $\mathrm{KSOM}$ in an incubator set to $37^{\circ} \mathrm{C}$ and $5 \% \mathrm{CO}_{2}$, after the sperm head was injected into the oocyte. PT androgenetic embryos were constructed following a previously described method [22]; however, our study used different electroporation parameters. We used two direct-current-pulses of $1.8 \mathrm{KV} / \mathrm{cm}$ for $10 \mu \mathrm{sec}$ each to complete cytoplast-karyoplasm fusion. In addition, DS androgenetic embryos were constructed following previously described methods [14, 23].

\section{Immunofluorescent staining and image analyses}

PT and DSandrogenetic embryos, as well as parthenogenetic embryos constructed by the maternal pronucleus, were transferred at $10 \mathrm{~h}$ post fertilization. The zona pellucida was dissolved using acidic CZB-HEPES solution. All samples were fixed in 4\% paraformaldehyde (Sigma, PFA, P6148) in phosphatebuffered saline (PBS) at room temperature for $40 \mathrm{~min}$. The samples were then permeabilized in $0.2 \%$ Triton X-100 in PBS at room temperature for $1 \mathrm{~h}$, after washing several times in HEPES-CZB. To observe $5 \mathrm{mC}$ and $5 \mathrm{hmC}$, permeabilized embryos were additionally placed in $2 \mathrm{~N} \mathrm{HCL}$ solution at room temperature for 50 min. The embryos were blocked with $1 \%$ bovine serum albumin (BSA) and $0.2 \%$ Triton $X-100$ in PBS overnight at $4{ }^{\circ} \mathrm{C}$. Then, the embryos were incubated with anti-Tet3 (monoclonal; Proteintech), anti$5 \mathrm{hmC}$ (polyclonal; Active Motif), and anti-5mC (monoclonal; Calbiochem) overnight at $4{ }^{\circ} \mathrm{C}$. The embryos were washed several times in $0.01 \%$ Tween 20 in PBS (PBST). They were then transferred to a secondary antibody mixture of Alexa Fluor 488 (anti-rabbit, Invitrogen) and Alexa Fluor 568 (anti-mouse, Invitrogen) at room temperature for $1 \mathrm{~h}$ in a dark place. After the nuclei were stained with $10 \mu \mathrm{g} / \mathrm{ml}$ Hoechst 33342, the embryos were mounted on slides with anti-fluorescence-fade medium (DABCO), and were examined by a laser-scanning confocal microscope (Zeiss, LSM700). In addition, we collected PT and DS blastocysts at $108 \mathrm{~h}$ post fertilization to examine the number of cells in the inner cell mass (ICM) and trophectoderm (TE) cells. Anti-OCT4 (monoclonal; Santa Cruz Biotechnology) for ICM and anti-CDX2 (monoclonal; Proteintech) for TE were used in this study. Tet3, $5 \mathrm{mC}$, and $5 \mathrm{hmC}$ dots were quantitatively analyzed with Image-pro plus version6.3.

\section{Western blotting}

To examine H3.3 protein expression in different androgenetic embryos, the male pronucleus from zygotes and double sperm heads-formed male pronucleus were collected. The female pronucleus from zygotes was treated as the control. We selected $10 \mathrm{~h}$ post fertilization as the examined time point. We used maternal pronucleus transfer (MT) embryos as the negative control. The same amounts of pronuclei were collected in each group. The number of pronuclei served as a reference in this experiment, rather than the Housekeeping gene. Twenty pronuclei from each group were placed in SDS sample buffer $(10 \mathrm{mmol} / \mathrm{L}$, $\mathrm{pH}$ 6.8, Tris-HCL, $20 \mathrm{mmol} / \mathrm{L}, 4 \% \mathrm{SDS}, 0.2 \%$ bromophenol blue, and $20 \%$ glycerol), and heated to $95^{\circ} \mathrm{C}$ for 5 min. Details of the procedure are provided in Zhao et al. [24]. The anti-H3.3 primary antibody (Rabbit 
polyclonal; Abcam) and the horseradish peroxidase-conjugated secondary antibody (Santa Cruz Biotechnology, USA) were used for this study. H3.3 signals were observed by the DAB detection system.

\section{Reverse transcription and quantitative PCR analysis}

The PT, DS, and ICSI embryos were collected at 78, 96, $108 \mathrm{~h}$ post fertilization as morulae, early blastocysts, and late blastocysts, respectively. mRNA from pools of 10 embryos was extracted using the Dynabeads mRNA DIRECT Micro Kit (Invitrogen, 61021), and were then used for reverse transcription with the High Capacity cDNA Reverse Transcription Kit (ABI, 4368814), following the manufacturer's instructions. Quantitative PCR (qPCR) was implemented using Top Green qPCR SuperMix (Transgen, AQ131) on a CFX96 Real-Time System (Bio-Rad, USA). The results were calculated using the $2 \triangle \triangle \mathrm{Ct}$ method. The expression of target CDNA was normalized to the expression of Gapdh. Primer sequences are listed in Table 1.

\section{Statistical Analysis}

The general linear models (GLM) procedure in the Statistical Analysis System (SAS User's Guide, 1985, Statistical Analysis System Inc., Cary, NC) was used to analyze the data from all the experiments. Significant differences were determined using Tukey's multiple range test. $\mathrm{P}<0.05$ was considered significant.

\section{Abbreviations}

PT Pronucleus transfer

DS Double sperm injection

ESCs Embryonic stem cells

ahESCs Androgenetic haploid ESCs

ZGA Zygote genome activation

5mC 5-methylcytosine

5hmC 5-hydroxymethylcytosine

ICM Inner cell mass

TE Trophectoderm

PMSG Pregnant mare serum gonadotropin 
hCG Human chorionic gonadotropin

PBS Phosphate-buffered saline

BSA Bovine serum albumin

PBST Tween-20 in PBS

GLM General linear models

SAS Statistical analysis system

\section{Declarations}

\section{Ethics approval and consent to participate}

All animal experiments were implemented in line with the Guidelines for Animal experiments of the Harbin Medical University (HMUIRB20190011). The approaches were approved by Harbin Medical University Ethics Committee.

\section{Consent for publication}

Not applicable

\section{Availability of data and materials}

All data generated or analyzed during this research are included in this published article.

\section{Competing interest}

The authors declare that they have no competing interest.

\section{Funding}

This study was supported by grants from National Natural Science Foundation of China (grant no. 31671545) and Overseas Scholars Research Funding Project of the Education Department of Heilongjiang Province (grant no. 1251H008). The funders had not contributed to the design of the research or in the collection, analysis or interpretation of results.

\section{Authors' contributions}


LC and SXH conceived the research, participated in its design, and wrote the manuscript. ZYW performed Western blot analysis and part of the immunofluorescence. LL participated in the conception of whole study and helped revise the article. All authors read and approved the final manuscript.

\section{Acknowledgements}

Not applicable

\section{References}

1. Kono $T$, Obata $\mathrm{Y}, \mathrm{Wu} \mathrm{Q}$, Niwa $\mathrm{K}$, Ono $\mathrm{Y}$, Yamamoto $\mathrm{Y}$, et al. Birth of parthenogenetic mice that can develop to adulthood. Nature. 2004;428(6985):860-4.

2. Li ZK, Wang LY, Wang LB, Feng GH, Yuan XW, Liu C, et al. Generation of Bimaternal and Bipaternal Mice from Hypomethylated Haploid ESCs with Imprinting Region Deletions. Cell Stem Cell. 2018;23(5):665-676.

3. Obata Y, Ono Y, Akuzawa H, Kwon OY, Yoshizawa M, Kono T. Post-implantation development of mouse androgenetic embryos produced by in-vitro fertilization of enucleated oocytes. Hum Reprod. 2000;15(4):874-80.

4. Keith E. Latham, Davor Solter. Effect of egg composition on the developmental capacity of androgenetic mouse embryos. Development. 1991;113(2):561-568.

5. Pan B, Yang H, Wu Z, Qazi IH, Liu G, Han H, et al. Melatonin Improves Parthenogenetic Development of Vitrified-Warmed Mouse Oocytes Potentially by Promoting G1/S Cell Cycle Progression. Int J Mol Sci. 2018;19(12).

6. Yanagimachi R. Male gamete contributions to the embryo. Ann N Y Acad Sci. 2005;1061:203-7.

7. Zhou LQ, Dean J. Reprogramming the genome to totipotency in mouse embryos. Trends Cell Biol. 2015;25(2):82-91.

8. Zheng W, Liu K. Maternal control of mouse preimplantation development. Results Probl Cell Differ. 2012;55: 115-39.

9. Li L, Zheng P, Dean J. Maternal control of early mouse development. Development. 2010,137(6):859-70.

10. Wen D, Banaszynski LA, Liu Y, Geng F, Noh KM, Xiang J, et al. Histone variant H3.3 is an essential maternal factor for oocyte reprogramming. Proc Natl Acad Sci USA. 2014;111(20):7325-30.

11. Lin CJ, Koh FM, Wong P, Conti M, Ramalho-Santos M. Hira-mediated H3.3 incorporation is required for DNA replication and ribosomal RNA transcription in the mouse zygote. Dev Cell. 2014;30(3): 26879.

12. Lin CJ, Conti M, Ramalho-Santos M. Histone variant H3.3 maintains a decondensed chromatin state essential for mouse preimplantation development. Development. 2013;140(17):3624-34. 
13. Gu TP, Guo F, Yang H, Wu HP, Xu GF, Liu W, et al. The role of Tet3 DNA dioxygenase in epigenetic reprogramming by oocytes. Nature. 2011;477(7366): 606-10.

14. Li W, Shuai L, Wan H, Dong M, Wang M, Sang L, et al. Androgenetic haploid embryonic stem cells produce live transgenic mice. Nature. 2012;490(7420):407-11.

15. Liu W, Yin j, Kou X, Jiang Y, Gao H, Zhao Y, et al. Asymmetric reprogramming capacity of parental pronuclei in mouse zygotes. Cell Rep. 2014;6(6):1008-16.

16. Boyer LA, Lee TI, Cole MF, Johnstone SE, Levine SS, Zucker JP, et al. Core transcriptional regulatory circuitry in human embryonic stem cells. Cell. 2005;122(6):947-56.

17. Scholer HR. Octamania: the POU factors in murine development. Trends Genet. 1991;7(10):323-9.

18. Hart AH, Hartley L, Ibrahim M, Robb L. Identification, cloning and expression analysis of the pluripotency promoting Nanog genes in mouse and human. Dev Dyn. 2004;230(1):187-98.

19. Deb K, Sivaguru M, Yong HY, Roberts RM. Cdx2 gene expression and trophectoderm lineage specification in mouse embryos. Science. 2006;311(5763): 992-6.

20. Géraud C, Koch PS, Zierow J, Klapproth K, Busch K, Olsavszky V, et al. GATA4-dependent organspecific endothelial differentiation controls liver development and embryonic hematopoiesis. J Clin Invest. 2017;127(3):1099-1114.

21. Salamone DF, Canel NG, Rodríguez MB. Intracytoplasmic sperm injection in domestic and wild mammals. Reproduction. 2017;154(6):F111-F124.

22. McGrath J, Solter D. Completion of mouse embryogenesis requires both the maternal and paternal genomes. Cell. 1984;37(1):179-83.

23. Zhao Q, Wang J, Zhang Y, Kou Z, Liu S, Gao SR. Generation of histocompatible androgenetic embryonic stem cells using spermatogenic cells. Stem Cells. 2010;28(2):229-39.

24. Zhao Q, Wu Y, Shan Z, Bai G, Wang Z, Hu J, et al. Serum starvation-induced cell cycle synchronization stimulated mouse rDNA transcription reactivation during somatic cell reprogramming into iPSCs. Stem Cell Res Ther. 2016;7(1):112.

\section{Tables}

Table 1 Primers Used for Real-Time PCR 


\begin{tabular}{llll}
\hline Genes & GenBank Accession No. & Forward & Reverse \\
\hline Gapdh & NM_008084 & AACTTTGGCATTGTGGAAGG & ACACATTGGGGGTAGGAACA \\
Ppia & NM_008907 & GAGCTCTGAGCACTGGAGAGA & CCACCCTGGCACATGAAT \\
& NM_013633 & & AGATGGTGGTCTGGCTGAAC \\
Oct4 & & CACGAGTGGAAAGCAACTCA & \\
Cdx2 & NM_007673 & TCTCCGAGAGGCAGGTTAAA & GCAAGGAGGTCACAGGACTC \\
Gata4 & NM_008092 & TGAAGGTCGGTGTGAACGGAT & CAGGGGGGCTAAGCAGTTGGT \\
Nanog & AY278951 & AAGTACCTCAGCCTCCAGCA & GTGCTGAGCCCTTCTGAATC \\
Casp3 & BC038825 & GGGCCTGTTGAACTGAAAAA & CCGTCCTTTGAATTTCTCCA \\
\hline
\end{tabular}

Table 2 Developmental rate of androgenetic embryos derived from pronucleus transfer or double sperm injection

\begin{tabular}{lllllll}
\hline & Total N & 2-cell (\%) & 4-cell (\%) & MO (\%) & BL (\%) \\
\hline ICSI & 481 & 8 & $432(89.9)$ & $364(84.4)$ & $319(73.8)$ & 298(68.9) \\
PT & 282 & 8 & $236(83.6)$ & $204(86.4)$ & $161(68.2)^{*}$ & $118(50.0)^{*}$ \\
\hline DS & 833 & 10 & $600(72.0)^{*}$ & $352(58.7)^{* *}$ & $148(24.7)^{* * *}$ & $46(7.6)^{* * *}$ \\
\hline
\end{tabular}

$\mathrm{N}$ : times of repetition; construction of one PT embryo needs two zygotes; PT, pronucleus transfer, DS, double sperm injection; $* P<0.05, * * P<0.01, * * * P<0.001$

Table 3 Cell numbers of androgenetic blastocysts of pronucleus transfer and double sperm injection

\begin{tabular}{llllll}
\hline & Number & Total & ICM & TE & Ratio (ICM/TE) \\
\hline ICSI & 22 & $58.39 \pm 13.1$ & $17.92 \pm 4.6$ & $40.46 \pm 9.9$ & $0.45 \pm 0.1$ \\
PT & 20 & $45.84 \pm 9.9^{*}$ & $13.42 \pm 5.1^{*}$ & $32.42 \pm 7.1^{*}$ & $0.42 \pm 0.1$ \\
\hline DS & 18 & $37.75 \pm 8.4^{* *}$ & $17.75 \pm 4.3$ & $20.0 \pm 7.5^{* *}$ & $0.89 \pm 0.1^{* *}$ \\
\hline
\end{tabular}

$\left({ }^{*} P<0.05 \square * * P<0.01\right)$

\section{Figures}


A MII Oocyte
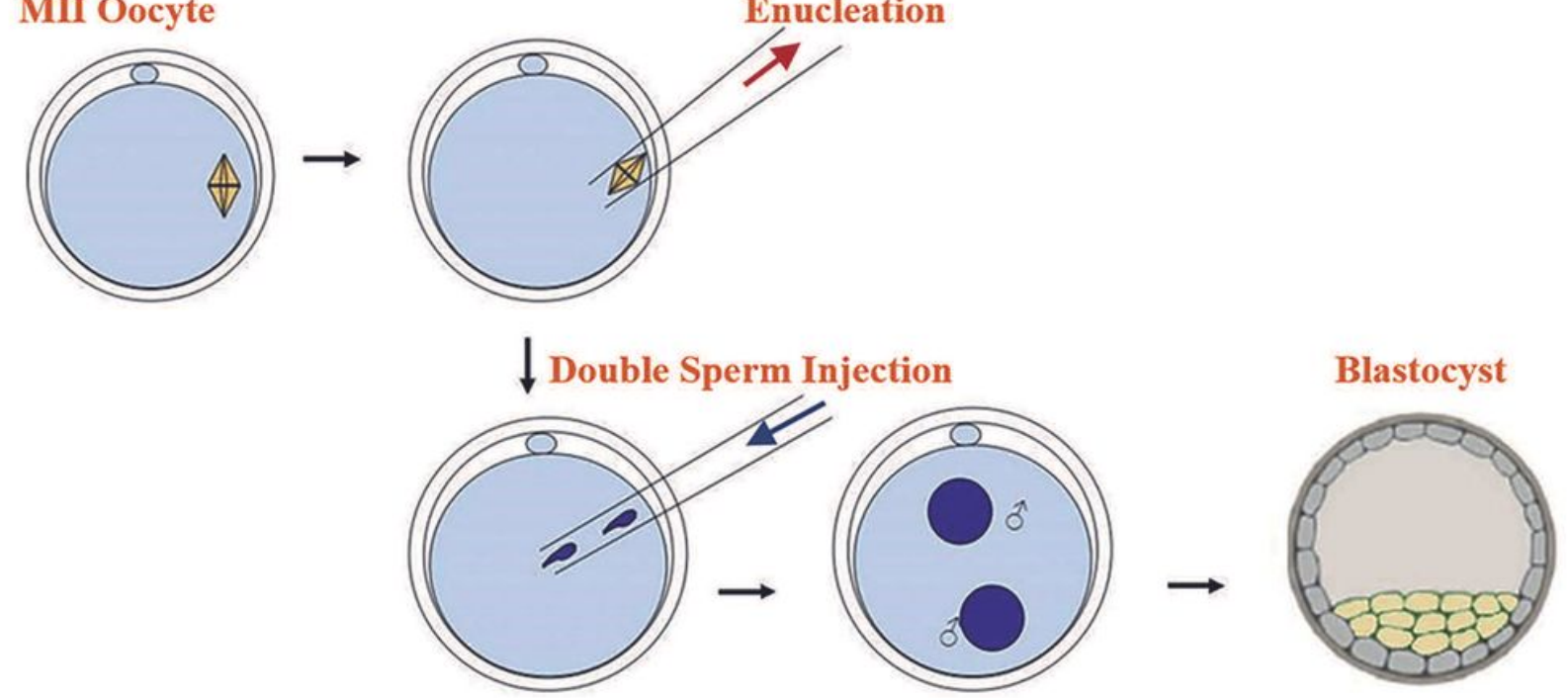

$\overline{\mathbf{B}}$
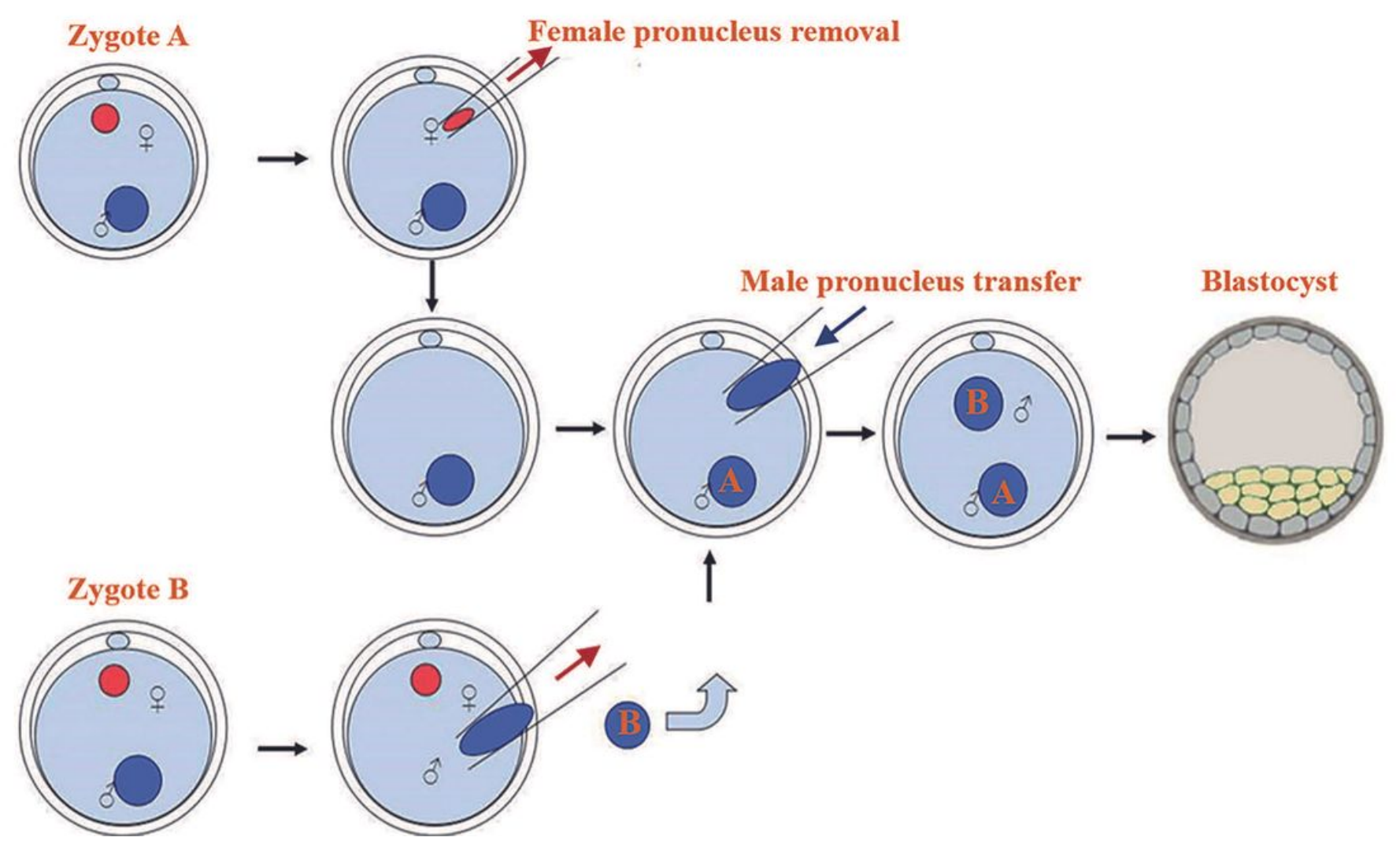

\section{Figure 1}

Schematic of androgenetic embryo construction. (A) Double sperm injection into cytoplasm of enucleated $\mathrm{M} \nabla$ oocyte. (B) Female pronucleus (smaller and closer to the polar body) is removed from zygote $A$; then, the male pronucleus from zygote $B$ is transferred to the perivitelline space. 


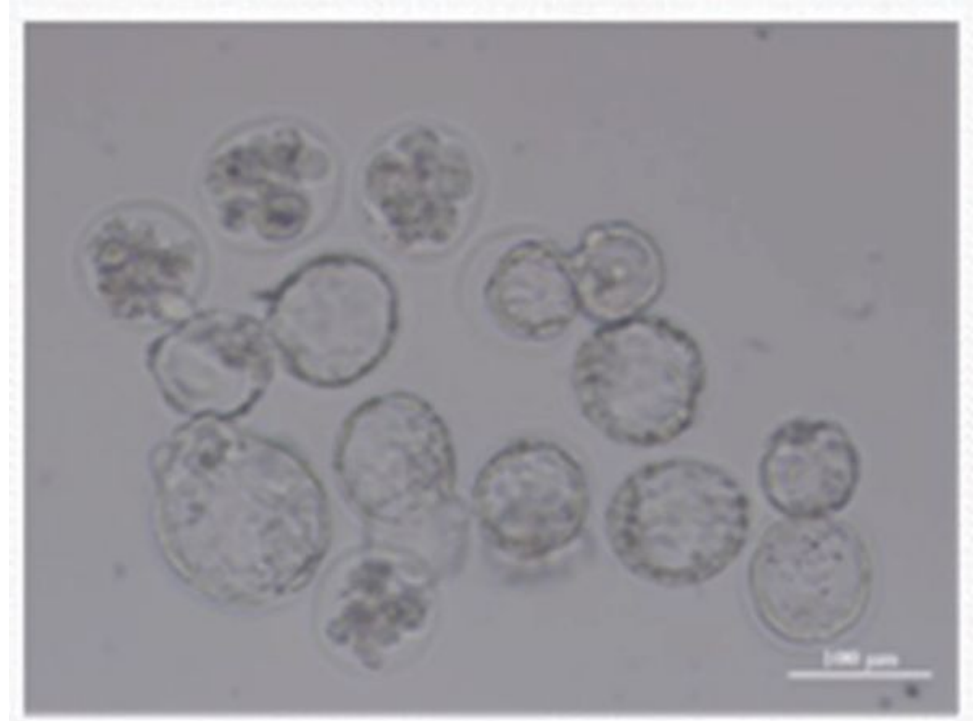

A. PT Blastocysts

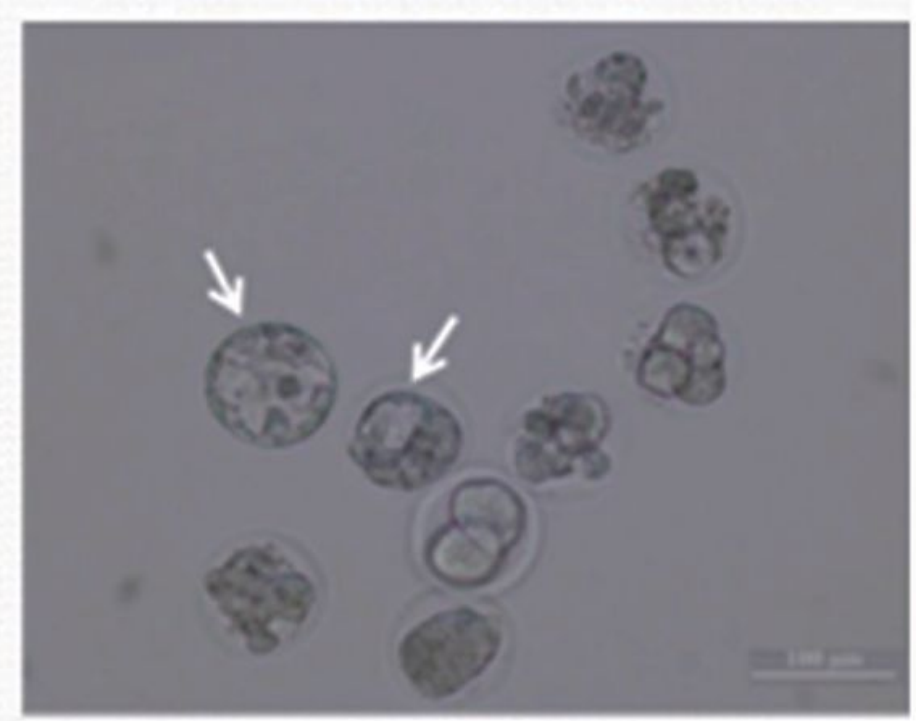

B. DS Blastocysts

\section{Figure 2}

Androgenetic blastocysts of pronucleus transfer and double sperm injection. (A) Androgenetic blastocysts of pronucleus transfer. (B) Androgenetic blastocysts of double sperm injection. The embryos, which develop into a blastocyst, were collected at $108 \mathrm{~h}$ post fertilization under a light microscope. (Scale: $100 \mu \mathrm{m}$; White arrows indicate blastocysts from the double sperm injection). 
A

M PN

DS PN

P PN

17KD

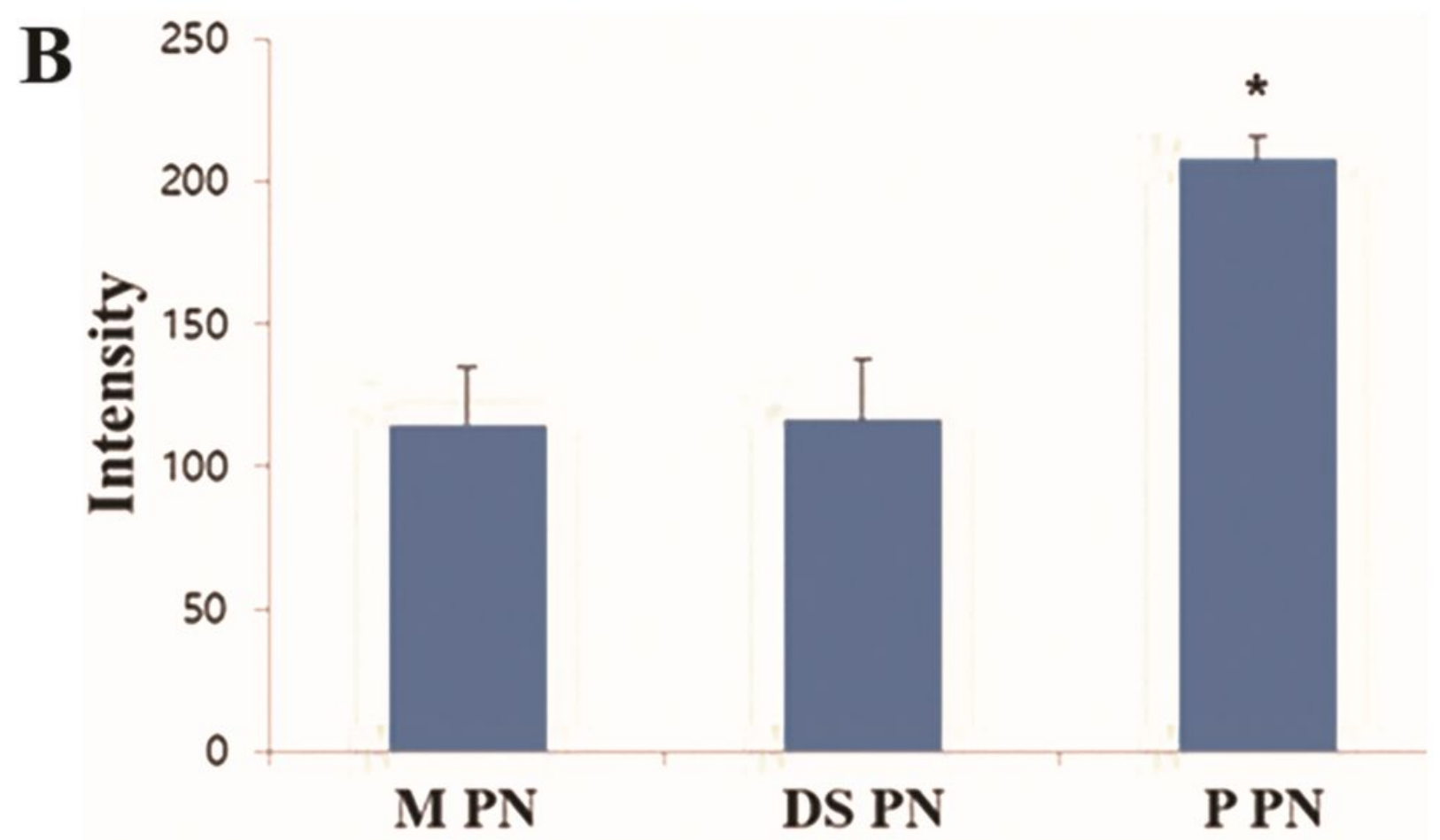

Figure 3

Expression of H3.3 in the pronucleus of androgenetic embryos. (A) H3.3 content in three groups of the pronucleus at $10 \mathrm{~h}$ post fertilization. M PN: Maternal pronuclei; DS PN, DS embryos' pronuclei; P PN paternal pronuclei. (B) Densitometry analysis of H3.3 protein ( ${ }^{*} \mathrm{P}<0.05$ ). (Because of undetectable amount of housekeeping protein in embryos, the same amounts of pronuclei were collected in each group. The number of pronuclei served as a reference in this experiment, rather than the housekeeping gene.) 

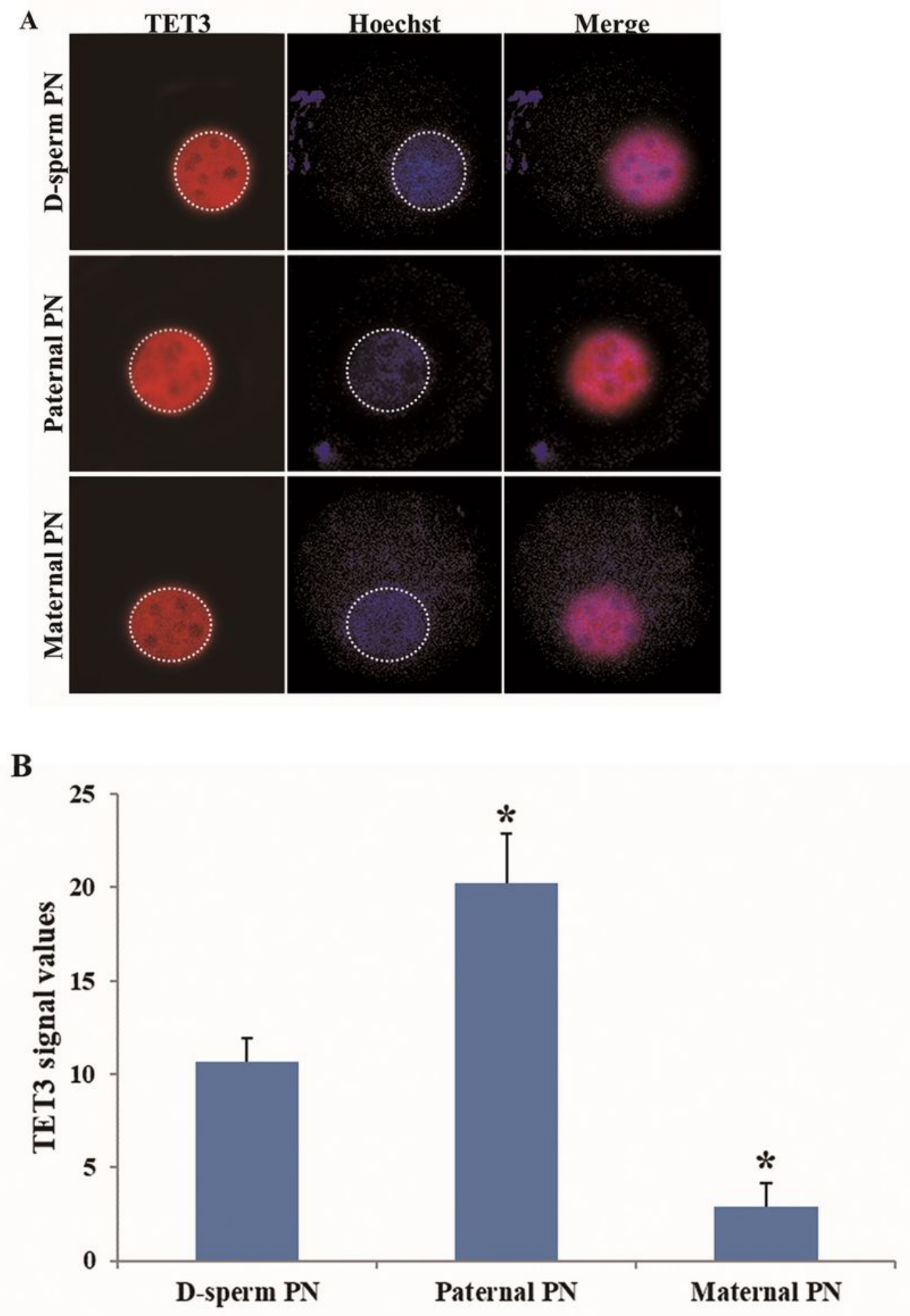

\section{Figure 4}

Detection of Tet3 expression through immunofluorescence double staining. (A) Level of Tet3 expression (red) at $10 \mathrm{~h}$ post fertilization. Blue: chromosome. (White circles indicate the region of the pronucleus; Dsperm PN: DS embryos' pronucleus; Paternal PN: PT embryos' pronucleus; Maternal PN: Maternal pronucleus. Scale: $50 \mu \mathrm{m}$ ). (B) Quantitative analysis of Tet3 fluorescence intensity in the pronuclei. There 
were at least 10 embryos in each group ( ${ }^{*} \mathrm{P}<0.05$ ). (Signal values reflect the fluorescence intensity in the pronucleus minus the fluorescence intensity in the cytoplasm).
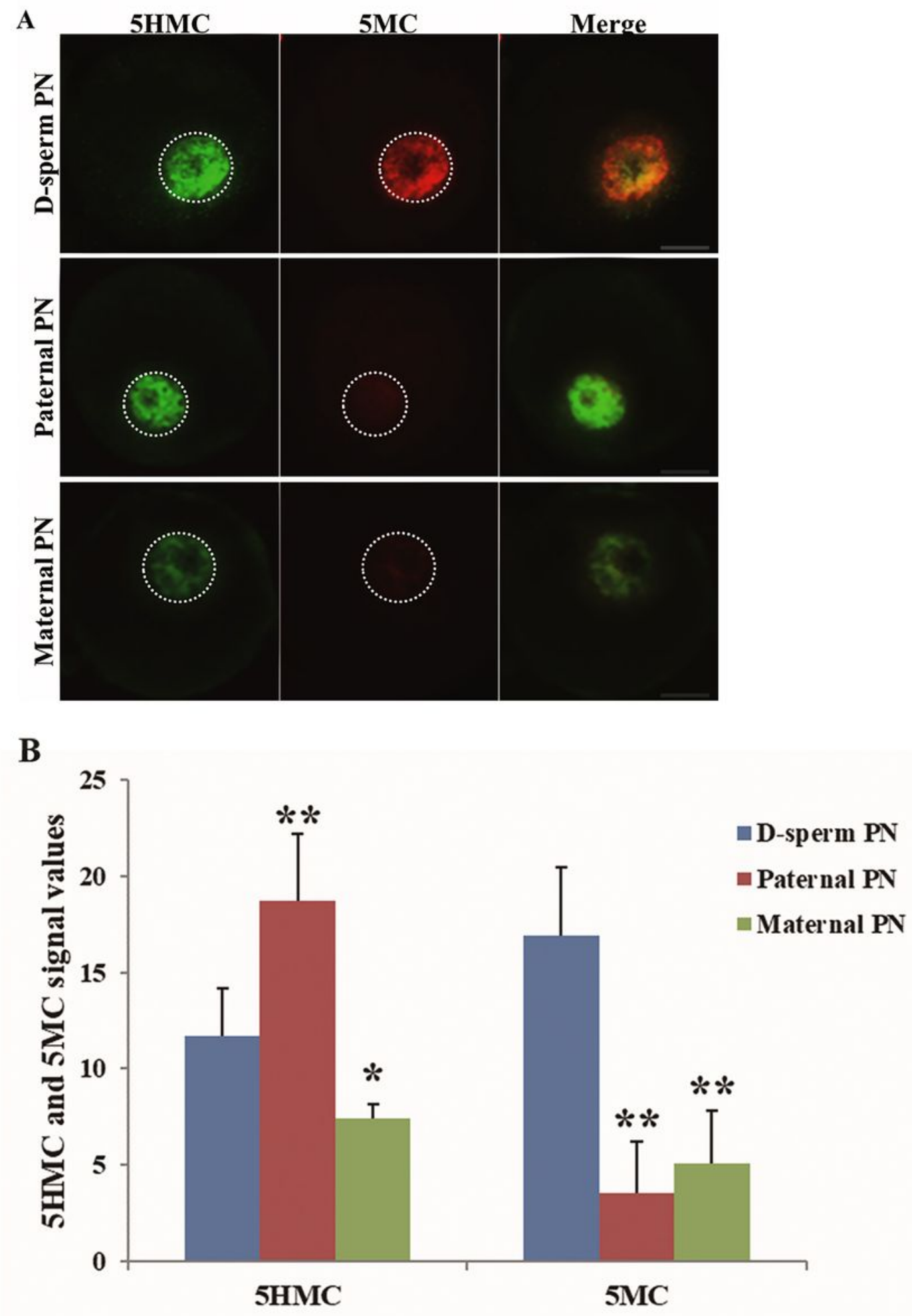

Figure 5

Detection of $5 \mathrm{mC}$ and $5 \mathrm{hmC}$ in the pronucleus. (A) $5 \mathrm{mC}$ and $5 \mathrm{hmC}$ levels at $10 \mathrm{~h}$ post fertilization. (White circles indicate region of pronucleus; D-sperm PN: DS embryos' pronucleus; Paternal PN: PT embryos' pronucleus; Maternal PN: Maternal pronucleus. Scale: $50 \mu \mathrm{m}$ ). (B) Quantitative analysis of fluorescence 
intensity of $5 \mathrm{mC}$ and $5 \mathrm{hmC}$ in the pronucleus. There were at least 10 embryos in each group $\left({ }^{\star} \mathrm{P}<0.05\right.$, $* * P<0.01)$.
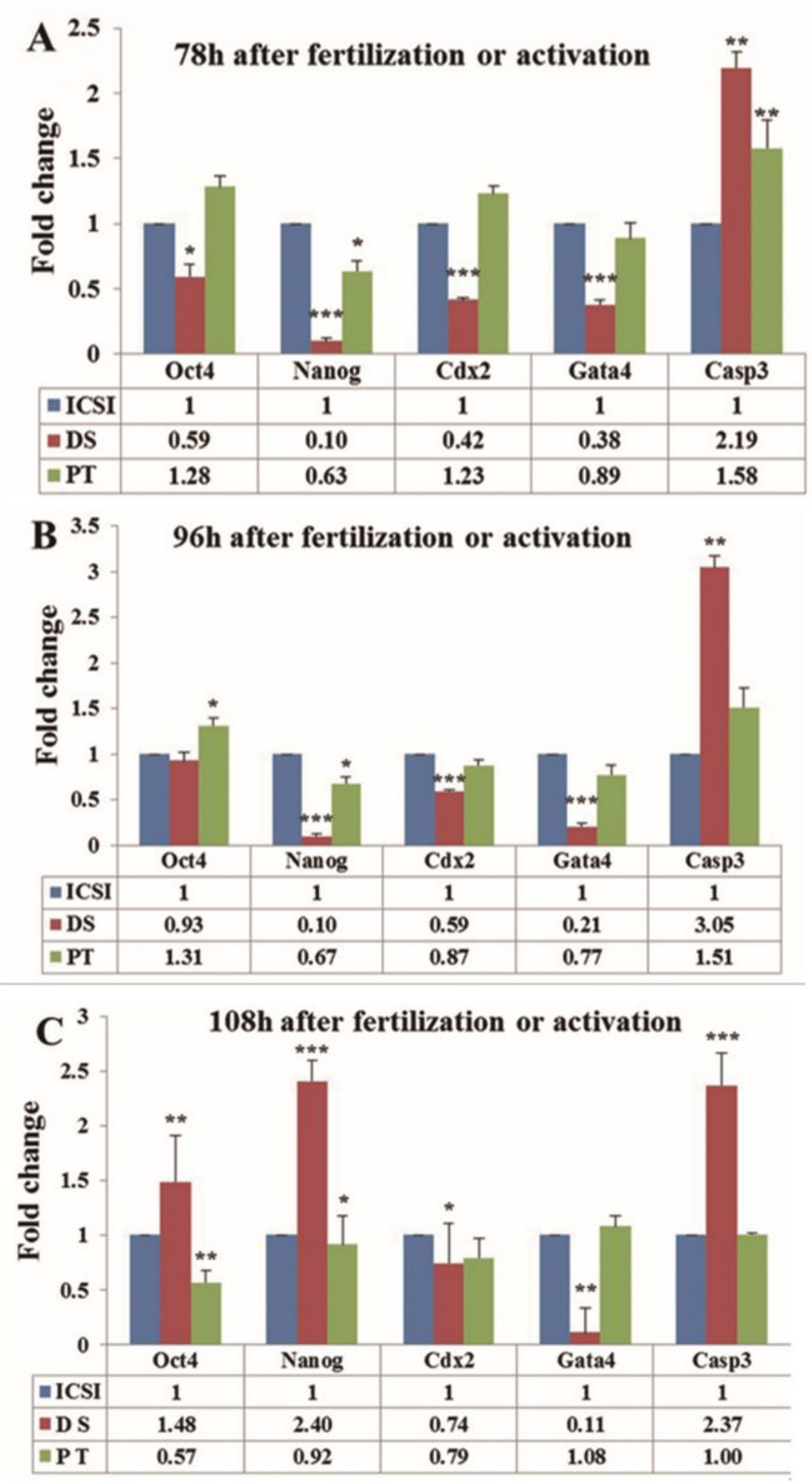

Figure 6

Detection of mRNA level of genes related to pluripotency and apoptosis, from the morula stage to the late blastocyst stage, in ICSI, DS, and PT androgenetic embryo using qPCR. (A) mRNA levels of Oct4, Nanog, Cdx2, and Casp3 at the morula stage. (B) mRNA levels of Oct4, Nanog, Cdx2, and Casp3 at the early 
blastocyst stage. (C) mRNA levels of Oct4, Nanog, Cdx2, and Casp3 at the late blastocyst stage $\left({ }^{\star} \mathrm{P}<\right.$ $\left.0.05,{ }^{* * \mathrm{P}}<0.01,{ }^{* \star *} \mathrm{P}<0.001\right)$.

\section{Supplementary Files}

This is a list of supplementary files associated with this preprint. Click to download.

- NC3RsARRIVEGuidelinesChecklist2014.docx1.docx 